\title{
Modern Medical Laboratory Journal
}

\section{The Prognostic Value of Serine and Glycine Levels in Plasma in Patients with Esophageal Cancer: A Case Control Study Mahsa Taherizadeh ${ }^{1}$, Masoud Khoshnia ${ }^{1}$, Sedigheh Shams ${ }^{2}$, Hamidreza Joshghani ${ }^{1 *}$}

1. Golestan Research Center of Gastroenterology \& Hepatology. Golestan University of Medical Sciences, Golestan, Gorgan, Iran

2. Children Medical center, Tehran University of Medical Sciences, Tehran, Iran

\begin{tabular}{c} 
KEYWORDS \\
\hline Esophageal Cancer; \\
Testis; \\
Serine; \\
Glycine; \\
HPLC \\
\\
\hline Article Info \\
\hline
\end{tabular}

Received 2019/02/23; Accepted 2019/03/05; Published Online 2019

\section{ABSTRACT}

Background and Objective: Serine and glycine are connecting lines for biosynthesis and are essential resources for synthesis of proteins, nucleic acids and lipids that are necessary for cancer cell growth. The purpose of this study was to set a comparison of serine and glycine in patients with esophageal cancer and in healthy people.

Materials and Methods: 37 plasma samples were collected from esophageal cancer patients and were referred to Gastrointestinal and Liver Disease Research Center, Firoozgar Hospital, affiliated to Iran University of Medical Sciences, Tehran, Iran. Plasma levels of branched-chain amino acids were measured by HPLC method. Statistics were calculated by SPSS v.16 software.

Results: In the patients' group the mean age \pm SD was $63 \pm 13.64$ and $21(56.8 \%)$ were male; while in control group, the mean age \pm SD was $64.24 \pm 13 / 08$ and $\% 54.1$ were male. Glycine levels were significantly increased in esophageal cancer $(P$ value: 0.031$)$ and age $(P$-value $<0.05)$ but it didn't have significant difference in association with sex $(P$-value $>0.05)$. However, serine levels in patients with esophageal cancer compared to the healthy group didn't show a significant difference $(P$-value: 0.610$)$ and also it didn't show a significant difference in association with age $(P$-value $>0.05)$, sex $(P$-value $>0.05)$.

Conclusion: High concentration of serine and reduced glycine levels in plasma of patients with esophageal cancer could act as a prognostic factor in cancer development.

Corresponding Information: Hamidreza Joahghani, Golestan Research Center of Gastroenterology \& Hepatology. Golestan University of Medical Sciences, Golestan, Gorgan, Iran. Email: joshaghani@goums.ac.ir. Tel: +98911 1779909

Copyright $\odot$ 2019. This is an open-access article distributed under the terms of the Creative Commons Attribution-noncommercial 4.0 International License which permits copy and redistribute the material just in noncommercial usages, provided the original work is properly cited.

\section{Introduction}

Esophageal cancer as a common malignancy of the elderly in which it has the highest rates at $7^{\text {th }}$ to $8^{\text {th }}$ decade of life, is a rare malignancy in young people. Esophageal cancer has two types of squamous cell carcinoma and adenocarcinoma based on pathological scales (1). Esophageal cancer in Iran has been under various investigations in different provinces and it revealed that in the northern and north eastern parts of Iran there is a higher rate of incidence $(2,3)$. Golestan province located in north east of Iran is one of the regions with higher frequency of esophageal cancer, whereas,
Mazandaran and Khorasan provinces are the next prevalent areas in Iran $(3,4)$.

More than one third of cancer patients are at risk of mortality after esophageal surgery and removing the malignant tissue due to cachexia $(5,6)$. It has been postulated that metabolism pathway alterations associated with malignancy, are the main key factor in patients' mortality. Actually, attempts to avoid cachexia by providing high amounts of protein or calorie have failed. In this regards, metabolism disorders in these patients should not be neglected ( 7 , 8). In carcinogenesis process, preserving cell growth 
and proliferation is associated with particular metabolism conditions $(9,10)$. Furthermore, these cells need higher energy and ingredients for new cellular components synthesis and also required as crucial factors to maintain redox and oxidation processes $(11,12)$.

Glucose and glutamine are two main sources for the activation of essential metabolic pathways such as glycolysis and Krebs cycle (TCA). In anabolic pathways, the serine synthesis is an important milestone in glucose conversion (13). Dietary serine and synthesized serine via glycolytic pathway can be converted to glycine and one-carbon units. The synthesis of proteins, lipids, nucleic acids, and other cofactors require one-carbon metabolism which is a complex metabolic network and takes place based on chemical reaction of folate compounds. One-carbon units are generated in some paths and are used in other metabolic pathways(14). Glycine produced from serine (serine hydroxymethyl transferase enzymes) that takes part in biosynthesis of Burin bases (two burin base carbon atoms and one nitrogen atom in burin ring). Glycine is an integral part of glutathione (the body's main antioxidant molecules) that is involved in cellular redox reactions. Also, glycine is consumed in mitochondria for biosynthesis, thus it plays a role in preserving oxidative phosphorylation. Recently, it has been shown that glycine uptakes and catabolism could promote tumorigenesis and cancer, and has been suggested that glycine metabolism could be considered as the main target for therapeutic interventions (15). The L-serine amino acid is one of the unnecessary nutrients (16)and it is produced by intracellular serine within 3-step enzymatic reaction of 3- Phosphoglycerate. Serine can also enter the cell by amino acid transporters from outside the cell(17). In addition to its role in protein synthesis, L-serine is used as prefabrication and essential metabolic intermediates for different biosynthetic pathways that include glycine synthesis, L- cysteine, phosphatidylserine (PS), sphingolipid and D Nmethyl-D- aspartate -serine activation (NMDA) and selective glutamate receptor. In addition, L-serine indirectly plays a role in purine and pyrimidine biosynthesis with transfer of a methylene group (C3serine) to tetrahydrofolate (THF)(18). One of the main metabolic pathways is converting serine to glycine which is done by serine hydroxymethyl transferase catalytic enzyme. The reaction catalyzed by serine hydroxymethyl transferase (SHMT) represents a major source of methyl groups for storing units of single carbon needed for biosynthesis of de novo nucleotides and DNA methylation. SHMT can be considered as a primary goal in two key paths for chemotherapy interventions: one is pathway of serine / glycine metabolism and the other is the biosynthesis of nucleotides. Serine can be converted to glycine in which, carbon units are provided for one-carbon metabolism.. One-carbon metabolism shows a complex metabolism which is based on chemical reactions of folate compounds. This path provides carbon for synthesis of proteins, lipids, nucleic acids and other cofactors. These single carbon units are the product of a cycle path and are transferred to other metabolic pathways(18). Thus, the aim of the present study was to investigate the metabolic abnormalities in order to study glycine and serine concentration in esophageal cancer.

\section{Material and Methods \\ Participants}

Plasma samples were taken from 37 patients with esophageal cancer referred to Gastrointestinal and Liver Disease Research Center, Firoozgar Hospital, affiliated to Iran University of Medical Sciences, Tehran, Iran and they were selected before any treatment (chemotherapy, radiation) and before surgery. Patients with other metastatic and metabolic diseases were excluded. Also, this study was confirmed by the Ethics Committee of Golestan University of Medical Sciences, Gorgan, Iran. The control group included healthy people, and those who did not have a history of familial cancer and malignant diseases. Written consent was obtained from each participant. All samples were collected from fasting patients. $5 \mathrm{ml}$ whole blood from all participants was collected in vacuum tubes containing EDTA and plasma was separated via centrifugation at $1000 \mathrm{~g}$ for 10 minutes and then they were kept frozen at $-80^{\circ} \mathrm{C}$.

\section{HPLC Measurement}

The analysis of serine and glycine amino acids was done by HPLC device model KNAUER (Germany). This study was conducted based on reverse-phase chromatography (RP-HPLC) and 
gradient method with flow rate as 1 and $\mathrm{ph}=7.02$ (19). In this step, 200 microliter sample was mixed with 50 microliter standard homoserine and 800 microliter methanol and incubated for $5 \mathrm{~min}$ at $4^{\circ}$ C. Then the supernatant was centrifuged for 5 minutes at $4000 \mathrm{rpm}$ and 250 microliter of it was mixed with 100 microliter borate buffer (keeping ph) was vortex for 5 seconds. Then 25 microliter normal HCL $75 \%$ was added to it and again 5 seconds vortex was done. Then, 50 microliter of the resulting solution was mixed with 200 microliter of solution A and 5 seconds vortex was done. After that 20 microliter of solution was taken by syringe Hamilton and injected to the HPLC device. Plasma levels of serine and glycine amino acids were measured by HPLC for 60 minutes.

\section{Statistics}

Dara were statistically analyzed using SPSS v.16 software by which standard deviation and mean concentration of serine and glycine were calculated.
$P$-values smaller than 0.05 were considered statistically significant, and t-test was selected for measuring any significant difference of plasma level of serine and glycine in patients with esophageal cancer and in healthy people.

\section{Results}

\section{Participants}

Out of 37 cases, the mean age \pm SD was $63 \pm 13.64$ and $21(56.8 \%)$ were male and out of 37 in the control group, the mean age \pm SD was $64.24 \pm 13.08$ and 54.1 were male. Glycine levels significantly increased in esophageal cancer $(P$ value: 0.031$)$, as age increased, it declined significantly too $(P$-value $<0.05)$, but it has not significant difference with sex $(P$-value $>0 / 05)$. Serine levels in patients with esophageal cancer compared to the healthy group was not significant ( $P$-value: 0.610$)$ also it was not significantly associated with Age ( $P$-value $>0.05)$, and sex $(P$ value $>0.05)$.

Table 1. Average concentration of serine and glycine in the case and control groups with t- test

\begin{tabular}{|c|c|c|c|}
\hline & & Serine $(\mu \mathrm{m})$ & Glycine $(\boldsymbol{\mu m})$ \\
\hline \multirow{2}{*}{ Case $(\mathbf{N}=37)$} & Mean \pm SD & $42.76 \pm 122$ & $89.19 \pm 260.62$ \\
\hline & Median & 125 & 245 \\
\hline \multirow{2}{*}{ Control $(\mathrm{N}=37)$} & Mean \pm SD & $48.22 \pm 123.15$ & $58.52 \pm 220.23$ \\
\hline & Median & 11.28 & 222 \\
\hline \multirow{3}{*}{$\begin{array}{c}\text { A comparison of } \\
\text { amino acids with test-t }\end{array}$} & p-value & 0.610 & 0.031 \\
\hline & Equal variances assumed & 0.934 & 0.934 \\
\hline & Equal variances not assumed & 0.025 & 0.025 \\
\hline
\end{tabular}

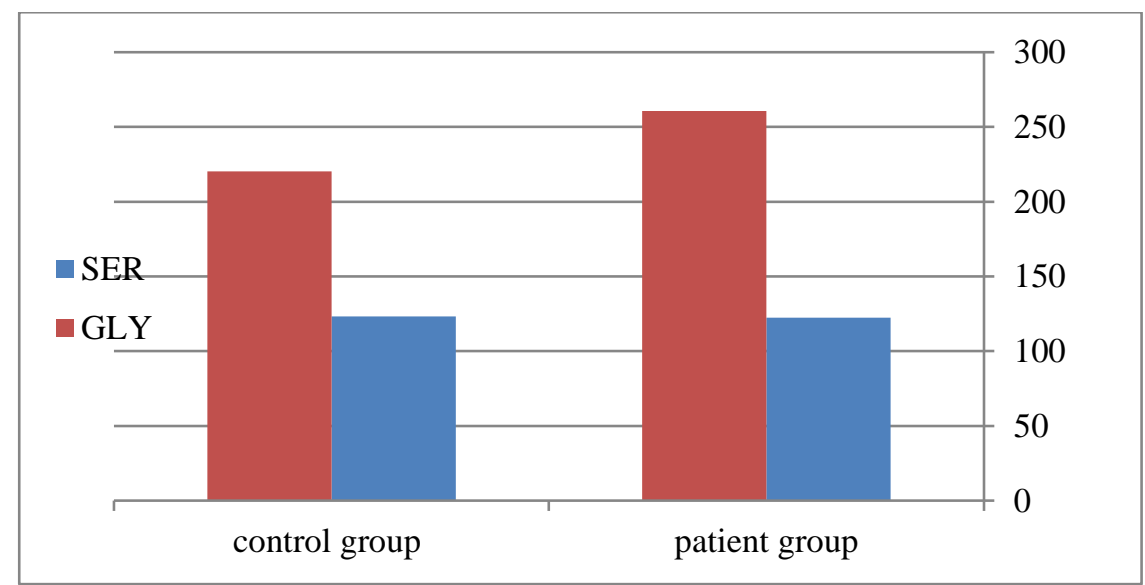

Fig. 1. Changes of serine and glycine levels in the patient group and the control group

\section{Discussion}


In the present study, we measured plasma concentrations of serine and glycine esophageal cancer patients and compared them with a control group. Results showed that serine concentration didn't have a significant difference and glycine concentration increased significantly in patients with esophageal cancer $(P$-value $<0.05)$.

Hong et al (20) reported that serine concentration significantly reduced and glycine significantly increased in esophageal cancer patients, also in this study glycine concentration was increased significantly. This increase might be due to the cellular biosynthesis potential and oxidation caused by using folate derivatives in one-carbon metabolism networks due to the cellular biosynthesis potential and oxidation and epigenetic status $(21,22)$. During the past 10 years, great efforts have been made on changes in metabolic properties of cancer cells(18). Now it is clear that with the Warburg effect, cancer cells increased flux through pentose phosphate pathway, high use of glutamine and through maintaining redox status (23, 24). By increasing lipid biosynthesis, cell proliferation in hypoxia and stress condition could be sustained. Also increased uptake of glycine and serine with cells has been observed (25).

Clarke et al(26) reported that the level of plasma Glycine increased and the results of our study confirmed this issue. Miyagi et al(27) showed an increase in plasma level of hreonine, serine, proline, glycine, alanine, ornithine and lysine, and a decrease in glutamine, tyrosine, histidine and tryptophan in breast cancer in comparison to the control group. In our study, results showed that the plasma level of Glycine in esophageal cancer was significant.

Recent works conducted at metabolomic cancer have shown that glycine metabolism is associated with the proliferation of cancer cells. In a study of cell panels NCI-60, the absorption and distribution rate of more than 200 metabolites was measured (15). Surprisingly, glucose uptake and lactate production (Warburg effect) is not related to cell proliferation and it is associated with cell proliferation through body metabolism. It is perceived that glycine uptake is strongly associated with cell proliferation (28). The over-expression of two enzymes involved in serine and glycine catabolism (phosphoserine aminotransferase and serine hydroxymethyl transferase) in NIH3T3 cells can induce tumor growth (28). The results of the study show that glycine plasma levels are variable in cancers.

\section{Conclusion}

Serine and glycine plasma changes in patients with esophageal cancer show that the conversion of serine to glycine metabolism increases in the presence of a malignant tumor. Data from this study is consistent with previous studies. In addition, studies have also shown that serine and glycine are produced in the body in patients with other cancers.

\section{Acknowledgement}

The laboratory which helped with the measurement of samples using HPLC is appreciated. Also all personnel who cooperated in the implementation and design of this study, evaluation of data and writing this paper are highly appreciated. Tehran Firoozgar Hospital and Research Center of Gastroenterology and Hepatology, Golestan Province are highly appreciated for cooperation in collecting samples.

\section{Conflicts of interest}

The authors state no conflicts of interest in this study.

\section{References}

1. Anil K. Rustgi MD aHBES MD, M.P.H. Esophageal Carcinoma. The New England Journal of Medicine. 2014;371:2499-509.

2. Islami F KF ND AK, Sotoudeh M, Abedi-Ardekani B, et al. Socio-economic status and oesophageal cancer: results from a populationbased case-control study in a high-risk area. Int JEpidemio. 2009;38:978-88.

3. R A. Five common cancers in Iran. Archives of Iranian medicine. 2010;13(2):143-6.

4. Ahmadi B, Alimohammadian M, Yaseri M, Majidi A, Boreiri M, Islami F, et al. Multimorbidity: epidemiology and risk factors in the Golestan cohort study, Iran: a cross-sectional analysis. 2016;95(7).

5. Talamonti MS KS YK, Wayne JD, Feinglass J, Bennett $\mathrm{CL}$, et al. Surgical outcomes of patients with gastric carcinoma: the importance of primary tumor location and microvessel invasion. Surgery. 2003;134(4):720-7.

6. Lyssiotis CA, Kimmelman ACJTicb. Metabolic interactions in the tumor microenvironment. 2017;27(11):863-75.

7. Vissers YL DC LY, Fearon KC, von Meyenfeldt MF, Deutz NE. Plasma arginine concentrations are reduced in cancer patients: evidence for arginine deficiency? The American journal of clinical nutrition. 2005;81(5):1142-6. 
8. Mayers JR, Vander Heiden MGJCr. Nature and nurture: what determines tumor metabolic phenotypes? 2017;77(12):3131-4.

9. VH M. Understanding the Warburg effect:the metabolic requirements of cell proliferation. Science. 2006;324:1029-33.

10. MT CRH. Regulation of cancer cell metabolism. Nat Rev Cancer. 2011;11(10):85-95.

11. AL SA. How cancer metabolism is tuned for proliferation and vulnerable to disruption. Nature reviews Cancer. 2012;491:364-73.

12. Ananieva EA, Wilkinson ACJCoicn, care m. Branchedchain amino acid metabolism in cancer. 2018;21(1):64.

13. RW KS. Resurgence of serine: an often neglected but indispensable amino Acid. Biol Chem. 2012;278 :19786-91.

14. LK FS. Temporary remissions in acute leukemia in children produced by folic acid antagonist, 4aminopteroyl-glutamic acid. $\mathrm{N}$ Engl J Med. 1948;238:787-93.

15. J M. Metabolite profiling identifies a key role for glycine in rapid cancer cell proliferation. Science. 2012;336:1040-44.

16. Furuya SJAPjocn. An essential role for de novo biosynthesis of L-serine in CNS development. 2008;17(S1):312-5.

17. Antonov A, Agostini M, Morello M, Minieri M, Melino $\mathrm{G}$, Amelio IJO. Bioinformatics analysis of the serine and glycine pathway in cancer cells. 2014;5(22):11004.

18. Ackerman D, Simon MCJTicb. Hypoxia, lipids, and cancer: surviving the harsh tumor microenvironment. 2014;24(8):472-8.
19. CJ TD. Rapid assay for amino acids in serum or urine by pre-column derivatization and reversed-phase liquid chromatography. 1982;23(8):527-31.

20. Hong Ma AH BM BK, Hai-Ping Zhang, Ilyar Sheyhidin. Plasma free amino acid profiling of esophageal cancer using high-performance liquid chromatography spectroscopy. World J Gastroenterol. 2014;20(26):8653-9.

21. Schulze A, Harris ALJN. How cancer metabolism is tuned for proliferation and vulnerable to disruption. 2012;491(7424):364.

22. Muñoz-Pinedo C, El Mjiyad N, Ricci JJCd, disease. Cancer metabolism: current perspectives and future directions. 2013;3(1):e248.

23. Lyssiotis CA V-HM M-PC EB. Emerging concepts: linking hypoxic signaling and cancer metabolism. Cell death \&disease. 2012;3:e303.

24. AD S. Hypoxia, lipids, and cancer:surviving the harsh tumor microenvironment. Trends in cell biology. 2014;24:472-8.

25. Amelio I CF AA AM, Melino G. Serine and glycine metabolism in cancer. Trends in biochemical sciences. 2014;39:191-8.

26. Clarke EF, Lewis AM, Waterhouse CJC. Peripheral amino acid levels in patients with cancer. 1978;42(6):2909-13.

27. Miyagi Y, Higashiyama M, Gochi A, Akaike M, Ishikawa $\mathrm{T}$, Miura $\mathrm{T}$, et al. Plasma free amino acid profiling of five types of cancer patients and its application for early detection. 2011;6(9):e24143.

28. L J. Serine, glycine and one-carbon units: cancer metabolism in full circle. Nature Reviews Cancer. 2013;13(8):572-83.

\section{How to Cite This Article:}

Taherizadeh M, Khoshnia M, Shams S, Joshaghani H. The Prognostic Value of Serine and Glycine Levels in Plasma in Patients with Esophageal Cancer: A Case Control Study. Mod Med Lab J. 2020; 3 (2) :69-73 